\section{Desvelando o cuidado às gestantes de alto risco em serviços de atenção primária do Município de São Paulo, Brasil: a ótica dos profissionais}

\section{Care for women with high-risk pregnancies in primary care services in the city of São Paulo, Brazil: the healthcare team's perspective}

\author{
Desvelando el cuidado a las gestantes de alto \\ riesgo en servicios de atención primaria del \\ Municipio de São Paulo, Brasil: la óptica \\ de los profesionales
}

Patricia Rodrigues Sanine 1 Sonia Isoyama Venancio 2 Fernanda Luz Gonzaga da Silva 3 Oswaldo Yoshimi Tanaka 3

\section{Resumo}

O presente estudo objetivou avaliar a atenção às mulheres durante a gestação de alto risco, sob a ótica de quem atua nos serviços de atenção primária à saúde (APS) do Município de São Paulo, Brasil. Trata-se de uma pesquisa avaliativa de abordagem qualitativa, que utilizou a técnica da entrevista aberta para a coleta dos dados com profissionais da APS. Os dados foram explorados por meio de categorias temáticas construídas a partir do conteúdo que emergiu, de modo espontâneo, dos depoimentos, discutidos a partir da Teoria do Processo de Trabalho em Saúde. Os resultados evidenciaram três categorias: uma "rotina de trabalho protocolar", organizada na perspectiva de práticas biologicistas e cujo modelo de gestão, algumas vezes, não permite o cumprimento das práticas preconizadas; um sistema de "referência e contrarreferência" que, por vezes, permite intervenções mais oportunas e singulares às necessidades das gestantes, mas, ainda, desarticulado dos serviços de referência; e a "corresponsabilização da equipe de APS pelo cuidado com a gestante”, caracterizado pela flexibilização da rotina de trabalho que transcende a aplicação de protocolos clínicos, incentivando a construção de vínculo e favorecendo a qualificação do cuidado. Desvelou-se a necessidade de flexibilização do uso de protocolos assistenciais no processo de trabalho, o que pode impulsionar a corresponsabilização das equipes de APS pelo cuidado, a fim de adequá-la às singularidades e necessidades de cada gestante, para além dos limites da organização dos serviços baseada na lógica de práticas biologicistas.

Gravidez de Alto Risco; Cuidado Pré-Natal; Atenção Primária à Saúde; Serviços de Saúde; Avaliação em Saúde

\author{
Correspondência \\ P. R. Sanine \\ Rua Carlos Guadanini 2406, Botucatu, SP 18610-120, Brasil. \\ patsanine@yahoo.com.br \\ 1 Faculdade de Medicina, Universidade Estadual Paulista, \\ Botucatu, Brasil. \\ 2 Instituto de Saúde, Secretaria de Estado da Saúde Pública de \\ São Paulo, São Paulo, Brasil. \\ 3 Faculdade de Saúde Pública, Universidade de São Paulo, São \\ Paulo, Brasil.
}




\section{Introdução}

As taxas de mortalidade materna e infantil são consideradas importantes indicadores que refletem condições socioeconômicas e de infraestrutura, mas principalmente da qualidade do sistema e dos serviços de saúde, em especial, os de atenção primária à saúde (APS), uma vez que apresentam atributos que os especificam com maior sensibilidade diagnóstica e alto poder resolutivo 1,2,3,4.

A importância destes serviços para a saúde materna e infantil é reconhecida desde a 5 a Conferência Nacional de Saúde, e sua capacidade de resposta às necessidades de saúde deste grupo fica evidente diante do impacto do aumento da cobertura populacional destes serviços na redução das taxas de hospitalização por doenças relacionadas ao pré-natal e parto, e da diminuição das iniquidades em saúde 5,6,7,8.

Várias iniciativas marcaram o fortalecimento da APS no Brasil, até à proposição atual da Estratégia Saúde da Família (ESF) como mecanismo de instaurar um modelo de cuidado mais efetivo 9,10,11,12. No caso específico do cuidado às mulheres durante a gestação de alto risco, que se apresenta como um período mais suscetível à ocorrência de complicações e óbito, a ideia de uma atenção efetiva avança da garantia do acesso para uma melhoria do processo de cuidado em saúde 5,13,14.

A qualidade é um termo polissêmico dentro do setor saúde, no entanto, considerou-se: acesso e atendimento precoce, acolhimento, referência e contrarreferência, além do cuidado compartilhado entre os serviços de APS e da atenção especializada 15.

Nesta perspectiva, ganha destaque a instituição da Rede Cegonha, que por meio da qualificação das ações à gestante e ao recém-nascido, simboliza uma importante estratégia em busca da garantia dos direitos das mulheres, além da ampliação do acesso, promoção da equidade e redução dos indicadores de mortalidade materna e neonatal 12,16.

No Estado de São Paulo, Brasil, observam-se iniciativas na busca pela melhoria, principalmente na capital, que implantou a Rede de Proteção à Mãe Paulistana, em 2006, integrando-a à Rede Cegonha, em 2011 13,17,18. Entretanto, isso ainda se apresenta como um desafio às políticas públicas estaduais $3,5,6,19,20,21$.

No Município de São Paulo, apesar de melhores do que a média brasileira, preocupa a elevada taxa de óbitos maternos tardios ${ }^{3}$, assim como a da incidência de mortalidade neonatal precoce e a de sífilis congênita 22 .

Este contexto, somado ao crescente cenário de política de austeridade e suas consequências sobre a saúde da população 2,6,23, evidenciam a relevância de estudos que busquem entender como ocorre a implementação dessas políticas no processo de trabalho em saúde pela visão de quem está na assistência.

Neste sentido, as abordagens qualitativas destacam-se por permitirem maior compreensão do contexto, pois por meio das falas e atitudes dos agentes sociais envolvidos no processo, têm o poder de captar todo o corpo de conhecimento proveniente de suas experiências e vivências, constituídas por valores, crenças e modos de pensar, sentir, se relacionar e agir 24 .

Considerando que as práticas de assistência à saúde são socialmente determinadas, transformando e sendo transformadas, dialeticamente, pelas necessidades sociais de saúde dos usuários e dos profissionais de saúde 25 , compreender a atenção às gestantes de alto risco pela ótica destes agentes promotores de saúde permite elucidar nossos questionamentos sobre quais fatores de contexto favorecem ou dificultam a qualidade desta atenção. Assim, objetivou-se avaliar a atenção às mulheres durante a gestação de alto risco, sob a ótica de quem atua nos serviços de APS de São Paulo.

\section{Métodos}

Pesquisa avaliativa de abordagem qualitativa, alicerçada em estudo de caso do tipo exploratório 26 , em serviços de APS do Município de São Paulo.

Entre as seis Coordenadorias Regionais de Saúde (CRS) do município, optou-se pela Zona Leste por ser uma das mais vulneráveis 27 : maior porcentagem de população sem plano de saúde $(67,8 \%$ em 2015); altos coeficientes de mortalidade infantil (13,4 por mil nascidos vivos) e neonatal precoce (6,0 por mil nascidos vivos), no ano de 2018; e de mortalidade materna (68,7 por 100 mil nascidos 
vivos), em 2017 22; além de apresentar grande diversidade de indicadores entre seus sete Distritos Administrativos (DA) 28.

Com uma área de $12.632 \mathrm{~km}^{2}, 2.467 .749$ habitantes e 112 serviços de APS, a Zona Leste possui diferentes coberturas entre os DA (19\% em Itaquera e 49,7\% em Itaim Paulista), além de grande diversidade no tempo médio de espera para consulta na atenção especializada, que em Itaim Paulista era de 156 dias, enquanto em Cidade Tiradentes era de 41 dias 28.

Para captar a diversidade de contextos, optou-se por uma amostra intencional a fim de apreender as distintas características da oferta de ações que permitissem analisar o alcance das práticas exercidas pelos serviços de APS, independentemente de seu modelo de atenção.

Buscando evitar viés de seleção, um dos critérios para escolha das unidades foi a incorporação dos diferentes modelos de atenção operacionalizados na APS da região: (1) USF (unidade com equipe de saúde da família exclusiva) e (2) UBS (unidade de saúde tradicional com ou sem assistência médica ambulatorial - AMA).

Entendendo que "qualidade" pressupõe o acesso das mulheres ao cuidado integral e que a articulação entre os diferentes níveis de atenção é um facilitador do alcance da integralidade, outro critério utilizado para a seleção dos serviços foi o nível de compartilhamento do acompanhamento do prénatal de alto risco entre os serviços de APS e atenção especializada: (1) alto (maioria das gestantes acompanhadas pelos dois serviços); (2) baixo (maioria acompanhada apenas na atenção especializada); e (3) intermediário (proporções semelhantes de gestantes que eram acompanhadas somente na APS e somente na atenção especializada).

Para tanto, partiu-se dos resultados de um inquérito conduzido no município para identificar o modelo de atenção e avaliar o funcionamento dos serviços de APS, além do compartilhamento do cuidado às gestantes de alto risco com a atenção especializada 20.

Atendendo a estes critérios, a amostra contou com seis serviços de APS, localizados em cinco DA da CRS Leste de São Paulo. A distribuição dos serviços conforme os critérios de seleção e algumas das suas características e contexto da região são apresentados na Tabela 1.

\section{Tabela 1}

Perfil da população residente na região e características dos serviços de atenção primária à saúde (APS) pertencentes à Coordenação Regional de Saúde Leste, Município de São Paulo, Brasil, conforme os critérios de seleção no ano de 2018.

\begin{tabular}{|c|c|c|c|c|c|c|c|c|}
\hline \multirow{2}{*}{$\begin{array}{l}\text { Distrito } \\
\text { Administrativo }\end{array}$} & \multicolumn{3}{|c|}{ Perfil da população } & \multicolumn{3}{|c|}{ Características dos serviços de APS } & \multicolumn{2}{|c|}{ Critério/Seleção } \\
\hline & $\begin{array}{c}\text { População } \\
\text { (habitantes) }\end{array}$ & $\begin{array}{c}\text { Partos no } \\
\text { SUS } \\
(\%)\end{array}$ & $\begin{array}{c}\text { Gestantes } \\
\text { adolescentes } \\
(\%)\end{array}$ & $\begin{array}{l}\text { Tempo de } \\
\text { espera na } \\
\text { APS (dias) }\end{array}$ & $\begin{array}{l}\text { Tempo de } \\
\text { espera na } \\
\text { atenção } \\
\text { especializada } \\
\text { (dias) }\end{array}$ & $\begin{array}{c}\geq 7 \\
\text { consultas } \\
\text { de pré- } \\
\text {-natal (\%) }\end{array}$ & $\begin{array}{c}\text { Nível de } \\
\text { acompanha- } \\
\text { mento entre } \\
\text { APS e atenção } \\
\text { especializada * }\end{array}$ & $\begin{array}{c}\text { Modelo de } \\
\text { atenção }\end{array}$ \\
\hline Ermelino Matarazzo & 208.254 & 66,1 & 10,9 & 33 & 79 & 48,2 & Intermediário & USF \\
\hline Guaianases & 279.146 & 72,9 & 14,2 & 36 & 110 & 43,8 & Alto & USF \\
\hline Itaim Paulista & 338.368 & 70,3 & 14,5 & 37 & 156 & 39,1 & Alto & UBS \\
\hline & & & & & & & Intermediário & UBS \\
\hline Itaquera & 546.280 & 65,4 & 12,0 & 45 & 99 & 52,7 & Baixo & USF \\
\hline São Mateus & 453.331 & 60,6 & 12,1 & 41 & 130 & 52,1 & Baixo & UBS \\
\hline
\end{tabular}

SUS: Sistema Único de Saúde; UBS: unidade de saúde tradicional com ou sem assistência médica ambulatorial - AMA; USF: unidade com equipe de saúde da família exclusiva.

Fonte: Sanine et al. 20.

* Alto: maioria das gestantes acompanhadas pelos dois serviços; Baixo: maioria acompanhada apenas na atenção especializada; e Intermediário: proporções semelhantes de gestantes que eram acompanhadas somente na APS e somente na atenção especializada. 
Considerando a Teoria do Processo de Trabalho em Saúde, na qual a dimensão organizacional do cotidiano dos serviços é definida a partir da práxis dos profissionais de saúde, que ao buscarem atender as necessidades do usuário, induzem novas práticas, ao mesmo tempo em que são induzidas por este consumo 25 , a produção de dados utilizou a técnica da entrevista aberta com agentes privilegiados neste processo.

Com a capacidade de captar uma informação que apenas quem vivenciou empiricamente a experiência pode fornecer 24,26 , compreendeu-se como informantes-chaves todas as categorias de profissionais envolvidas na atenção às gestantes de alto risco.

Foram entrevistados um profissional por categoria, envolvido diretamente no atendimento às gestantes e disponível no período das entrevistas, totalizando 27 profissionais: seis médicos, seis enfermeiros, seis reguladores de vagas para a atenção especializada, seis gerentes dos serviços, e três agentes comunitários de saúde (ACS), categorias presentes apenas nas USF.

O dia e horário das entrevistas foram definidos previamente e ocorreram dentro do ambiente de trabalho dos participantes, durante o mês de junho de 2018. Todos autorizaram a gravação em áudio e assinaram o Termo de Consentimento Livre e Esclarecido (TCLE), conforme aprovação no CAAE: 43716515.7.1001.5404 da Universidade Estadual de Campinas e da Faculdade de Saúde Pública, Universidade de São Paulo.

Utilizou-se uma abordagem de livre expressão, o que possibilitou uma narrativa espontânea por parte dos participantes sobre sua atuação frente ao objeto de estudo 26 . Buscando imparcialidade, não se mencionou a questão do alto risco, iniciando as entrevistas pela solicitação: "Conte sobre seu trabalho com as gestantes neste serviço de APS”, seguida de estímulos na busca por detalhes, sem nenhuma pergunta que caracterizasse algum grau de indução nas respostas.

As entrevistas foram realizadas e transcritas na mesma semana, por dois dos autores e um colaborador externo. Os serviços foram numerados de 1 a 6 e os participantes de 1 a 5 . Assim, os depoimentos foram identificados pela composição de dois números, por exemplo: 1.1 (...) 6.5.

A exploração dos dados foi realizada por meio de categorias temáticas construídas a partir do conteúdo que emergiu, de modo espontâneo, dos depoimentos. Conforme proposto por Minayo 24, a metodologia constou de uma primeira aproximação do conteúdo, buscando identificar as abordagens relatadas em relação à atenção às gestantes de alto risco, seguido de leitura aprofundada, destacando aqueles que se repetiam, ou que precisavam ser confirmados por outras falas. Nesta etapa, identificaram-se unidades de significado que foram agrupadas conforme suas semelhanças, construindo uma primeira dimensão empírica.

A partir do aprofundamento da análise, estas unidades de sentido foram reagrupadas, permitindo desvelar as categorias-chave no atendimento às gestantes de alto risco em serviço de APS que, discutidas à luz da Teoria do Processo de Trabalho em Saúde 25, favoreceram a compreensão do contexto e do quanto estas práticas atendem às necessidades de saúde postas para este grupo.

A sistematização das categorias de análise utilizou o programa Microsoft Word (https://products. office.com/) e foi iniciada por um dos autores que participou de todo processo, além de discutida com os demais, totalizando um período de seis meses.

\section{Resultados e discussão}

A abordagem utilizada possibilitou desvelar a complexidade dos contextos envolvidos no processo de trabalho das equipes de APS em relação à atenção às gestantes de alto risco. O conteúdo das entrevistas foi agrupado em três categorias: uma "rotina de trabalho protocolar", organizada na perspectiva de práticas biologicistas e cujo modelo de gestão, algumas vezes, não permite o cumprimento das práticas preconizadas; um sistema de "referência e contrarreferência" que, por vezes, permite intervenções mais oportunas e singulares às necessidades das gestantes, ainda que desarticulado dos serviços de referência; e a "corresponsabilização da equipe de APS pelo cuidado com a gestante", caracterizado pela flexibilização da rotina de trabalho que transcende a aplicação de protocolos clínicos, incentivando a construção de vínculo e favorecendo a qualificação do cuidado. 
Identificou-se uma rotina de trabalho protocolar, caracterizada pela definição de diferentes atribuições aos profissionais da equipe e a utilização de normativas, como, por exemplo, a definição do número de consultas e formas de encaminhamento para outros níveis de atenção:

"Com gestante nosso trabalho consiste em fazer seguimento, os controles que têm como base um protocolo de 6 [consultas] (...), o que vamos fazer em cada avaliação médica, os exames" (1.1).

"Depois de aberto o sistema do Mãe Paulistana, a enfermeira segue todo o protocolo (...) Depois de 30 dias a gestante vai para o médico, ai ele vai clinicar e ver se ela é de alto risco. Passa primeiro pelo médico, preenche o formulário e vai para o alto risco" (5.2).

"É assim, a gestante de alto risco não passa com a enfermagem, só passa com o médico, então é só comigo. O seguimento é assim: é lá [na atenção especilizada] e aqui" (3.1).

"Todos os encaminhamentos vão para regulação e precisam vir com o nome da especialidade, CID [Classificação Internacional de Doenças] e carimbo médico" (4.4).

A realização do primeiro atendimento à gestante com solicitação de exames e agendamento de consulta médica, no dia em que a mulher é notificada do resultado do teste de gravidez, demonstrou a existência de uma rotina que favorece o acesso ao acompanhamento pré-natal 14.

Do mesmo modo, os depoimentos alinham-se às recomendações sobre a garantia de um número mínimo de consultas e do acompanhamento do pré-natal de alto risco ser centralizado no atendimento médico. Por outro lado, o vínculo da mulher durante a gestação com toda a equipe de saúde, também, é um determinante para o adequado resultado no acompanhamento pré-natal 3,14,29.

Algumas práticas que buscam a garantia de acesso e de atendimento para um número maior de gestantes, como a definição da duração de 15 minutos das consultas em todos os serviços, podem desfavorecer outras dimensões do cuidado, mostrando-se insuficiente para atender às necessidades postas pelas gestantes de alto risco:

"A gente conversa um pouquinho somente durante a anamnese, que analisa sintomatologia, peso, altura, pressão arterial, altura uterina, batimento cardíaco fetal e, se ela tiver outra queixa, a gente avalia. Se nessa avaliação for identificado algum grau de risco gestacional, tem alguns critérios de encaminhamento..." (6.1).

"...É muito arriscado, porque são 5 minutos para arrumar as coisas, que são compartilhadas. Fica só 10 minutos para atendimento e se ela me fala de outro problema, como que passou no convênio ou na $A E$ [atenção especializada], eu preciso dar atenção. Não tem como alcançar o tempo [estipulado]” (1.1).

"A gente faz muito mais consultas do que a prevenção e promoção em saúde (...) é mais rotina, tem aquele 4 por hora - 15 minutos cada consulta" (3.1).

Depoimentos como estes revelam limitações técnico-operacionais influenciadas pelo modelo de gestão da APS que, por vezes, tem como principal foco o cumprimento de metas de produtividade, ao mesmo tempo em que impõe uma rotina protocolar às equipes que desconsidera, muitas vezes, as próprias políticas - inclusive municipais 18,30,31.

Esta influência do modelo de gestão sobre as práticas de saúde exercidas nos serviços de APS, fortemente associada à sua administração por convênios com Fundações e/ou Organizações Sociais de Saúde 32, foi reforçada pelo depoimento de que:

"Algumas gestantes têm interesse por planejamento familiar, mas a nossa referência de laqueadura é um hospital com função religiosa, que não opera. Então, não temos para onde encaminhar. Mas acabamos conversando com uma enfermeira de outro hospital e mandando casos muito limítrofes, pois como não temos essa referência, eles acabam, eventualmente, cedendo uma ou duas vagas no ano" (5.3).

O fato de somente o médico acompanhar as gestações de alto risco e, consequentemente, em tempo reduzido, acaba por acumular uma preocupação em cumprir as práticas preconizadas, além de ofertar menos tempo de consulta comparado às gestantes de risco habitual (que são atendidas, também, pelos enfermeiros).

Este tipo de cobrança culmina na produção de um trabalho sem investigação ou busca ativa de sinais e sintomas que não foram relatados pela gestante, resultando em uma prática mecânica, focada no modelo biologizante de cuidado e, portanto, pouco resolutiva 8,18,30,31.

“...Uma gestante que acompanha só com o ginecologista, carrega várias dúvidas ao longo da gestação. Não que o médico não saiba responder, mas não está focado em ensinar a pessoa a ter um autocuidado. Eu entendo esta orientação como a parte-chave do cuidado" (2.2). 
Este pouco contato da gestante de alto risco com o profissional enfermeiro(a) que, também é responsável pelo acompanhamento do recém-nascido e da puérpera após sua alta na maternidade, fragiliza a construção de um vínculo, o que pode comprometer a integralidade do cuidado e, consequentemente, a qualidade da atenção:

“...A gestante do alto risco nem avisa quando nasceu o bebê, porque faltou vínculo. Ela ficou tendo visita do ACS e atendimento com o médico, que tem uma visão mais clínica, mas a visão dos cuidados, da orientação, que é do enfermeiro, não teve. Quando o bebê nasce a gente tem que conhecer o ambiente em que essa criança vai ser inserida, mas como você entra na casa de uma pessoa que não te viu a gestação inteira?” (1.2).

A fala do ACS também mostra uma desarticulação da rede de atenção às mulheres durante a gestação de alto risco, com falhas no sistema de referenciamento da maternidade para a APS após a alta hospitalar, ainda que este seja enfatizado na Rede Cegonha 16. Essa desarticulação pode impactar de forma negativa a continuidade do cuidado da mulher pelo serviço de APS e o fortalecimento do vínculo com a equipe, além de gerar expectativas de que a mulher se encarregue dessa comunicação com a APS.

Por outro lado, é possível constatar um avanço em relação à presença de um profissional de regulação de vagas dentro do próprio serviço de APS, responsável pelos encaminhamentos das gestantes de alto risco para a atenção espaecializada. Isso favorece o alcance das recomendações preconizadas no protocolo municipal, que estabelece que o tempo de espera entre o encaminhamento da APS e a consulta na atenção especializada não deve ultrapassar 30 dias.

"Em alguns casos que vieram de outra região, esconderam a gestação, ou que têm a idade já avançada, fazemos esse contato com a referência para conseguir captar rapidamente essa vaga, pra não dar 30 dias porque, assim, ficamos mais tranquilos quanto àquela gestação" (2.3).

Assim, a referência e contrarreferência caracterizou-se como uma categoria temática que vai além da implementação de uma rotina de trabalho protocolar, com circulação de papéis e encaminhamentos 8 , mas que ao inserir o regulador de vagas, mostrou favorecer uma intervenção mais voltada às necessidades das gestantes, facilitando um funcionamento que valoriza as singularidades, como ao permitir que quando não se encontra a vaga disponível para uma gestante que apresente situações de maior risco, o mesmo modifique as prioridades de agendamento.

Vários depoimentos retrataram esta preocupação do regulador em entender a real necessidade da mulher, elucidando o quanto algumas destas situações, além de oportunizarem o acesso, se mostraram relacionadas à construção de um vínculo entre equipe e gestante, ao mesmo tempo em que sinalizam uma responsabilização pelo cuidado:

“...Eu costumo dizer que ali nós somos quase psicólogos, porque temos que ouvir o paciente, ver a necessidade dele e correr atrás do que ele precisa. (...) procuramos encaminhar aqueles com os casos mais agravantes" (3.4).

"Descrevo o caso do paciente e encaminho para eles [atenção especializada], porque aí seria uma alta prioridade que eu consigo dar" (1.4).

"Tenho que ficar monitorando essas vagas para ver se saiu porque não chegam avisos. Por exemplo, tem vagas com CID de câncer, aí eu tento ver todos os dias porque pode perder a vaga. Tem que tomar cuidado, pode sair de um dia para o outro" (2.4).

Falas como estas mostram como a presença do regulador pode favorecer a identificação das necessidades de cada gestante e o acesso em tempo oportuno à atenção especializada, evitando a demora do atendimento que pode impactar em desfechos desfavoráveis. No entanto, falhas no encaminhamento, aliadas à falta de contrarreferência aos outros níveis de atenção, corroboram com situações que evidenciam baixa qualidade do cuidado, favorecendo a peregrinação das gestantes na procura por maternidade no momento do parto 19,23,33.

"Às vezes a própria obstetra do alto risco manda alta da paciente e explica que não tem o critério para acompanhar lä" (2.1).

"A resolutividade da paciente que foi encaminhada é no hospital. Na maioria das vezes, ela retorna contando verbalmente o que aconteceu. Nunca temos uma contrarreferência” (5.3).

Considerando a gravidade da situação provocada pelos encaminhamentos inadequados e pela falta de contrarreferência, somada à limitação de acesso associada às características individuais das gestantes 20,23, o desenvolvimento de ações que fortaleceram o vínculo, representado pela corresponsabilização da equipe de APS pelo cuidado 14 destacou-se como uma prática que potencializa uma atenção qualificada: 
"Acho que o vínculo que tentamos criar com a gestante é atender da melhor maneira possivel. Deixar as portas sempre abertas, explicar todo o procedimento, o porquê estamos pedindo aqueles exames (...) muitas pessoas esperam anos para poder engravidar e quando engravidam, a gente tem que tratar não o 100\%, mas o $1.000 \% "(6.2)$.

Desta forma, a corresponsabilização da equipe de APS pelo cuidado com a gestante foi identificada nas falas dos entrevistados como uma dimensão proativa direcionada à flexibilização da rotina de trabalho protocolar e à construção do vínculo.

Caracterizada pela identificação e resolução de problemas e sintomas identificados, como ao antecipar a realização de exames em casos de maior gravidade ou ao fazer procedimentos não inclusos na rotina do serviço, esta categoria, também, reforça que a qualificação do cuidado às gestantes de alto risco transcende a aplicação de protocolos clínicos:

"Só encaminhar e deixar ela só lá [na atenção especializada], não dá certo. Até porque, às vezes, ela não vai conseguir passar lá todos os meses, mas aqui consegue, porque é perto. E se pudermos, adiantamos alguns exames, para ela chegar lá com eles prontos, porque é muita paciente no alto risco e facilita se já tiver alguma coisa mais pronta" (2.1).

"Você fica preocupado [com gestantes faltantes], então tratamos de agendar em um horário que seja bom ou falamos para a gestante vir o horário que ela quiser. Porque tem gestante que não vem por problema do trabalho, então damos um jeitinho" (1.1).

Pontuando que os protocolos, mais do que regras, referem-se à diretrizes organizacionais e de conduta e que, buscando maior efetividade, possibilitam modificações na execução das atividades 31 , pode-se observar que este comportamento dos profissionais resultou na adequação das práticas exercidas na rotina dos serviços, proporcionando uma articulação em um âmbito mais amplo.

“Tem muita coisa que a gente não pode fazer (...), mas vou encaminhar para cirurgia que demora? (...) A unha já está ruim, então avaliamos um jeito, falamos com o dentista para usar materiais dele e faço assim mesmopara o bem do paciente" (1.1).

“...A partir do momento que identifico que Fulana faltou, já tenho o costume de já na hora, pelo Whatsapp da equipe, já falar: 'Fulana de tal faltou e pede para ela vir tal data”' (3.3).

Reforça, ainda, a importância da integração e valorização de todos os membros da equipe, incluindo o ACS, assim como do fortalecimento da atuação da equipe multiprofissional e a articulação com profissionais da atenção especializada.

"Procuramos ter esse vínculo com o médico e com o enfermeiro para justamente ter facilidade [de encaixe]. Às vezes vem uma demanda, aí conversamos em reunião e decidimos o melhor. Se um está com dúvida, os mais velhos dão uma força” (6.5).

“...Alguns anos atrás o farmacêutico ficava preso na farmácia, hoje ele participa de reunião técnica discutindo fluxo, protocolo, entrando na reunião de categoria médica para orientar sobre medicação ou mudanças dos protocolos. E tem em contrapartida, médicos que têm essa abertura, que anotam um bilhetinho pedindo para convocar quando a gestante falta" (2.3).

“Às vezes a profissional de saúde serve até como psicóloga. (...) Às vezes eles desabafam com a gente. Fico muito satisfeita porque a pessoa tem confiança (...) às vezes levo o assunto para o NASF [Núcleo de Apoio à Saúde da Família], para a psicóloga fazer uma terapia em grupo ou uma consulta individual, se ela não quer falar em público" (3.5).

Nesta perspectiva, é possível constatar o quanto a corresponsabilização pelo cuidado apresentase como um qualificador da atenção, ao mesmo tempo em que favorece a construção do vínculo. Em contrapartida, a existência de vínculo entre equipe e gestante, e sua articulação com outros serviços, apresenta-se, também, como um fator que fortalece o cuidado qualificado.

Em outras palavras, perceber a necessidade momentânea da usuária, logo no acolhimento ou nas ações de busca ativa, proporcionando flexibilidades no atendimento, foi o fator que permitiu o verdadeiro acesso desta mulher ao sistema, reconhecendo que ela é muito mais do que uma gestante de alto risco.

O modo de acolher as demandas e construir um vínculo foram os principais diferenciais para a qualidade da atenção, sinalizado pela coordenação e pela longitudinalidade do cuidado presentes na vinculação da gestante na APS, ou seja, a manutenção do seu acompanhamento mesmo quando encaminhada para a atenção especializada: 
"O relato que eu tenho hoje, que não é nada oficial, é que elas [gestantes de alto risco] vão para lá [atenção especializada], mas que elas querem continuar aqui” (6.3).

Ao compreender o cuidado às gestantes de alto risco como o resultado da atenção integral compartilhada entre os profissionais da APS e da atenção especializada 5,14,20, evidenciou-se que o cuidado com responsabilidade está localizado nas ações que fazem com que estas sejam percebidas pelos usuários como acesso oportuno e atenção às suas expectativas de cuidado, ao mesmo tempo em que constrói um bom vínculo com a equipe de saúde.

Este reconhecimento do serviço como local que irá atender as necessidades da mulher e ofertar um cuidado qualificado à gestante de alto risco, ficou explicitado na postura proativa de alguns profissionais, principalmente dos serviços do tipo USF, cujas falas se mostraram sensíveis à percepção destas necessidades individuais das gestantes, comprometidos com a equipe em ofertar um cuidado diferenciado:

"Se ela procura, é porque está precisando. Então, vamos dar uma atenção a mais. Agora, se é um paciente que nunca procura, quando ele procura, a gente dá uma atenção maior. Tentamos resgatá-lo de todas as formas, seja no encaixe, ou na visita domiciliar" (6.5).

"Esta confiança que você ganha, muitas vezes, faz com que você possa compartilhar mais coisas. E dependendo da paciente, se tiver alto risco, eu fico preocupado porque se algo acontece com ela, eu sei que a responsabilidade é de todos" (1.1).

Pode-se perceber, nestes depoimentos, que o termo acesso à atenção, apesar de bastante empregado à acessibilidade, se refere a um conceito amplo, que representa muito mais do que um "fenômeno material e físico" traduzido pela entrada do usuário ao serviço 34.

Corroborando este resultado, estudos apontam o acolhimento com a prática de escuta qualificada das queixas para a identificação de demandas reprimidas e esclarecimento das dúvidas das gestantes como importantes dispositivos da Política Nacional de Humanização (PNH), representando uma tecnologia essencial para a qualidade da atenção ao pré-natal e puerpério 8,35,36.

Assim, tais resultados desvelaram que, muito mais do que a "recepção do usuário no serviço", o vínculo deve ser estimulado como incremento para o acesso e melhoria do processo de trabalho, representando a corresponsabilização do profissional pelo acompanhamento e a (re)orientação do cuidado pela rede de atenção ${ }^{8}$.

Como já referenciado pela literatura 20,37, a atuação específica atribuída ao ACS junto à equipe de APS, especialmente relacionada às visitas domiciliares, se destacou como fator positivo neste processo:

"Meu trabalho é fazer a visita, ver se o paciente foi na consulta, se está precisando de alguma coisa, se tiver algum evento na unidade, eu convido. Se eu percebo que tem alguém que não está passando em consulta, insisto para que ele venha até a unidade. Meu trabalho é praticamente a preocupação com os pacientes" (6.5).

"Muitas vezes, elas [gestantes adolescentes] descobrem que estão grávidas porque o ACS que orienta" (1.2).

"Nós [ACS] montamos uma apresentação para levar para a comunidade de uma forma lúdica o que é a Estratégia [Saúde da Família] e o trabalho do ACS” (2.5).

Posturas como as apresentadas, aumentam o poder de utilização do serviço pelas gestantes e favorecem a construção do vínculo, que oportuniza o cuidado qualificado 19:

"A grande maioria delas vivem em uma situação vulnerável e a gente precisa ir à casa para ver o que está acontecendo e não perder este vínculo" (2.1).

"[A visita domiciliar] é muito importante, porque os exames que a AE [atenção especializada] passar, provavelmente, ela vai realizar aqui e às vezes ela esquece de agendar ou demora para procurar o serviço [APS]" (6.5).

Ao mesmo tempo, tais resultados somam-se a estudos que enfatizam a "necessidade de maiores esforços dos municípios para incentivar a inclusão dos ACS” 38 (p. 606) nos serviços de APS e nos processos avaliativos. A Política Nacional de Atenção Básica (PNAB), ao retirar a obrigatoriedade de um número mínimo de ACS, flexibilizando sua contratação conforme o grau de vulnerabilidade do local ao qual o serviço está inserido, busca incentivar a inserção destes profissionais. Mas cabe pontuar que permite, também, a redução de tais admissões 10.

Tendo em vista que o cuidado em saúde se concretiza pelo processo de trabalho que se transforma em ato pela interação entre o profissional de saúde e o usuário 25 , convém salientar iniciativas pessoais e/ou coletivas dos profissionais da APS, que como particularidades individuais dos participantes, nem 
sempre podem ser consideradas replicáveis, mas que, no entanto, se destacaram positivamente em relação à corresponsabilização das equipes pelo cuidado e, consequentemente, para a qualidade da atenção às gestantes de alto risco:

"Quando se trata de gestação de alto risco a paciente passa com o médico daqui e o da especialidade, mas isso não impede que nós, enfermeiras, façamos uma visita domiciliar ou até mesmo uma consulta” (3.2).

"Se o médico achar que é um caso de urgência, faz uma cartinha do próprio punho e a gente encaminha para o regulador" (2.4).

"Quando tem algum problema eu falo assim: '(...) Fora do horário de trabalho só consigo dar orientação por celular, porque não posso ir até a casa dela"' (1.1).

"Por isso que eu falo que tem que andar com essa carteirinha de pré-natal no bolso, porque tudo vai estar escrito ali. (...) Acontece alguma coisa, a culpa é da Maternidade ou do parto que demorou, mas às vezes, é uma patologia. Por isso que prezo por um pré-natal bem feito e uma carteirinha com letra legivel” (4.1).

"Conhecemos o pessoal da referência de ultrassom que às vezes conseguem um encaixe. Conversando com eles fora do padrão de fila de espera para tentar suprir a necessidade" (1.4).

Tais resultados reforçam que para a qualidade do cuidado às gestantes de alto risco não basta esta prática convencional. Parece que a preocupação em atender a esta rotina pré-estabelecida por metas, alimentam uma padronização do cuidado que aliena os profissionais de saúde com um processo de trabalho burocrático e, portanto, distante de uma assistência reflexiva e comprometida com a responsabilização pelo cuidado, como na flexibilização do trabalho.

Os resultados apresentados foram capazes de desvelar alguns dos fatores contextuais que podem favorecer ou dificultar a qualidade da atenção às gestantes de alto risco, como o modelo de gestão e, principalmente, de atenção operacionalizada nos serviços refletidos, por exemplo, na presença dos agentes comunitários de saúde e do regulador de vagas, na cobrança por produtividade que reduz o tempo de consulta e "engessa" o atendimento. Em que pesem as limitações metodológicas do estudo que não contemplou outros informantes (como gestores e usuários dos serviços) e a restrição para apenas uma região do Município de São Paulo -, a identificação de tais resultados evidenciam o poder de replicação do método, ao mesmo tempo em que permite levantar novas hipóteses, que podem ser melhor aprofundadas em outras análises, como em relação à influência do perfil das categorias profissionais e dos modelos de atenção operacionalizados nestes serviços.

\section{Considerações finais}

O esforço em avaliar o processo de trabalho pela ótica de quem realiza o cuidado, mais do que saber se os serviços estão cumprindo os protocolos, permitiu desvelar as variáveis de contexto diferenciais para favorecer a qualidade do cuidado.

Identificou-se a importância dos protocolos assistenciais na rotina de trabalho das equipes, porém, faz-se necessário propiciar a flexibilização nas práticas e ampliar o olhar do cuidado oferecido, buscando responder às expectativas e necessidades das mulheres. Esta adaptação das práticas impulsionou a corresponsabilização da equipe de APS pelo cuidado, mesmo diante do encaminhamento da gestante à atenção, favorecendo a construção do vínculo e qualificação do cuidado.

Ao buscar atender essas necessidades, ou antever outros riscos, potencializou-se a integralidade e longitudinalidade do cuidado, atributos fundamentais neste nível de atenção. Neste mesmo sentido, as fragilidades identificadas na articulação com os serviços da rede acabam sendo minimizadas com a inserção do regulador de vagas, que permite intervenções mais oportunas e singulares às necessidades das gestantes.

Por fim, reforça-se que o grande potencial está na melhor utilização dos protocolos e de sua flexibilização no processo de trabalho. Enfatiza-se a necessidade de uma prática diferenciada, que considere as singularidades e necessidades específicas de cada gestante, captadas em uma escuta ampliada e cuidadosa, para além dos limites da organização dos serviços baseada na lógica de práticas biologicistas, que se mostram insuficientes para enfrentar a complexidade posta para a atenção às mulheres durante a gestação de alto risco. 


\section{Colaboradores}

P. R. Sanine participou da elaboração da proposta e todas as demais etapas de preparação do artigo. S. I. Venancio colaborou na análise e redação final do texto. F. L. G. Silva participou das entrevistas, transcrições, análise e revisão final do texto. O. Y. Tanaka colaborou na elaboração da proposta, análise e revisão final do texto.

\section{Informações adicionais}

ORCID: Patricia Rodrigues Sanine (0000-00027668-0327); Sonia Isoyama Venancio (0000-00017147-3292); Fernanda Luz Gonzaga da Silva (00000002-2576-458X); Oswaldo Yoshimi Tanaka (00000002-5653-0794).

\section{Agradecimentos}

Os autores agradecem a colaboração da Coordenadoria Regional de Saúde Leste/São Paulo e aos profissionais dos serviços de atenção primária à saúde, além da pesquisadora Lídia Pereira da Silva Godoi, que colaborou nas entrevistas e transcrições. Ao Ministério da Ciência Tecnologia e Inovações, Conselho Nacional de Desenvolvimento Científico e Tecnológico e Ministério da Saúde pelo financiamento (MCTI/CNPq/MS - SCTIE - DECIT no 41/2013).

\section{Referências}

1. Rede Interagencial de Informação para a Saúde. Indicadores básicos para a saúde no Brasil: conceitos e aplicações. Brasília: Rede Interagencial de Informação para a Saúde; 2008.

2. Rasella D, Basu S, Hone T, Paes-Sousa R, Ocké-Reis CO, Millett C. Child morbidity and mortality associated with alternative policy responses to the economic crisis in Brazil: a nationwide microsimulation study. PLoS Med 2018; 15:e1002570.

3. Vega CEP, Soares VMN, Nasr AMLF. Mortalidade materna tardia: comparação de dois comitês de mortalidade materna no Brasil. Cad Saúde Pública 2017; 33:e00197315.

4. Victora CG, Aquino EML, Leal MC, Monteiro CA, Barros FC, Szwarcwald CL. Maternal and child health in Brazil: progress and challenges. Lancet 2011; 377:1863-76.

5. Rehem TCMSB, Ciosak SI, Egry EY. Internações por condições sensíveis à Atenção Primária no hospital geral de uma microrregião de saúde do município de São Paulo, Brasil. Texto Contexto Enferm 2012; 21:535-42.

6. Guimarães WSG, Parente RCP, Guimarães TLF, Garnelo L. Acesso e qualidade da atenção pré-natal na Estratégia Saúde da Família: infraestrutura, cuidado e gestão. Cad Saúde Pública 2018; 34:e00110417.

7. Viacava F, Oliveira RAD, Carvalho CC, Laguardia J, Bellido JG. SUS: oferta, acesso e utilização de serviços de saúde nos últimos 30 anos. Ciênc Saúde Colet 2018; 23:1751-62.

8. Feuerwerker LCM. Micropolítica e a política e a gestão em saúde. In: Feuerwerker LCM, organizador. Micropolítica e saúde: produção do cuidado, gestão e formação. Porto Alegre: Editora Rede Unida, 2014. p. 63-118. (Coleção Micropolítica do Trabalho e o Cuidado em Saúde)

9. Cecilio LCO, Reis AAC. Apontamentos sobre os desafios (ainda) atuais da atenção básica à saúde. Cad Saúde Pública 2018; 34:e00056917.

10. Morosini MVGC, Fonseca AF, Lima LD. Política Nacional de Atenção Básica 2017: retrocessos e riscos para o Sistema Único de Saúde. Saúde Debate 2018; 42:11-24.

11. Portela GZ. Atenção primária à saúde: um ensaio sobre conceitos aplicados aos estudos nacionais. Physis (Rio J.) 2017; 27:255-76.

12. Ministério da Saúde. Implantação das Redes de Atenção à Saúde e outras estratégias da SAS. Brasília: Ministério da Saúde; 2014.

13. Sanine PR, Castanheira ERL. Explorando nexos entre a construção social da criança e as práticas de saúde. Hist Ciênc Saúde-Manguinhos 2018; 25:199-215.

14. Secretaria Estadual da Saúde de São Paulo. Atenção à gestante e à puérpera no SUS - SP: manual técnico do pré-natal e puerpério. São Paulo: Secretaria Estadual da Saúde de São Paulo; 2010

15. Ministério da Saúde. Gestação de alto risco: manual técnico. Brasília: Ministério da Saúde; 2012. 
16. Ministério da Saúde. Manual prático para implementação da Rede Cegonha. Brasília: Ministério da Saúde; 2011.

17. Secretaria Municipal da Saúde de São Paulo. Relatório Rede de Proteção à Mãe Paulistana também é Rede Cegonha. São Paulo: Secretaria Municipal da Saúde de São Paulo; 2015.

18. Mota A, Schraiber LB. Atenção primária no sistema de saúde: debates paulistas numa perspectiva histórica. Saúde Soc 2011; 20:837-52.

19. Fernandes JA, Venâncio SI, Pasche DF, Silva FLG, Aratani N, Tanaka OY, et al. Avaliação da atenção à gestação de alto risco em quatro metrópoles brasileiras. Cad Saúde Pública 2020; 36:e00120519.

20. Sanine PR, Venancio SI, Silva FLG, Aratani N, Moita MLG, Tanaka OY. Atenção ao pré-natal de gestantes de risco e fatores associados no Município de São Paulo, Brasil. Cad Saúde Pública 2019; 35:e00103118.

21. Sanine PR, Zarili TFT, Nunes LO, Dias A, Castanheira ERL. Do preconizado à prática: oito anos de desafios para a saúde da criança em serviços de atenção primária no interior de São Paulo, Brasil. Cad Saúde Pública 2018; 34:e00094417.

22. Secretaria Estadual de Saúde de São Paulo. TabNet. https://www.prefeitura.sp.gov. $\mathrm{br} / \mathrm{cidade} / \mathrm{secretarias} / \mathrm{saude} / \mathrm{tabnet} / \mathrm{index}$. php? $\mathrm{p}=6522$ (acessado em 08/Jun/2019).

23. Mendes RB, Santos JMJ, Prado DS, Gurgel RQ, Bezerra FD, Gurgel RQ. Características maternas e da assistência pré-natal associadas à peregrinação no anteparto. Rev Saúde Pública 2019; 53:70.

24. Minayo MCS. Análise qualitativa: teoria, passos e fidedignidade. Ciênc Saúde Colet 2012; 17:621-6.

25. Paim JS. Da teoria do processo de trabalho em saúde aos modelos de atenção. In: Ayres JRCM, Santos L, organizadores. Saúde, sociedade e história. São Paulo: Hucitec Editora; 2017. p. 375-92.

26. Yin RK. Estudo de caso: planejamento e métodos. Porto Alegre: Bookman; 2015.

27. Bugni RP, Jacob MS. Índice de vulnerabilidade social: uma análise da cidade de São Paulo. In: Margutti BO, Costa MA, Favarão CB, organizadores. Territórios em números: insumos para políticas públicas a partir da análise do IDHM e do IVS de UDHs e regiões metropolitanas brasileiras. Rio de Janeiro: Instituto de Pesquisa Econômica Aplicada; 2017. p. 117-50.
28. Secretaria Municipal da Saúde de São Paulo. Boletim CEInfo Saúde em Dados 2018; Ano XVII, no 17.

29. Neves RG, Flores-Quispe MDP, Facchini LA, Fassa AG, Tomasi E. Pré-natal no Brasil: estudo transversal do Programa de Melhoria do Acesso e da Qualidade da Atenção Básica, 2014. Epidemiol Serv Saúde 2020; 29:e2019019.

30. Moraes PND, Iguti AM. Avaliação do desempenho do trabalhador como forma peculiar de prescrição do trabalho: uma análise do PMAQ-AB. Saúde Debate 2013; 37:416-26.

31. Righi LB. Apoio matricial e institucional em saúde: entrevista com Gastão Wagner de Sousa Campos. Interface (Botucatu) 2014; 18 Suppl 1:1145-50.

32. Sanine PR, Dias A, Machado DF, Zarili TFT, Carrapato JFL, Placideli N, et al. Influência da gestão municipal na organização da atenção à saúde da criança em serviços de atenção primária do interior de São Paulo, Brasil. Cad Saúde Pública 2021; 37:e00242219.

33. Troncon J, Quadros Netto DL, Rehder PM, Cecatti JG, Surita FG. Mortalidade materna em um centro de referência do Sudeste Brasileiro. Rev Bras Ginecol Obstet 2013; 35:388-93.

34. Frenk JO. El concepto y la medición de acessibilidad. Salud Pública Méx 1985; 27:439-53.

35. Garuzi M, Achitti MCO, Sato CA, Rocha SA, Spagnuolo RS. Acolhimento na Estratégia Saúde da Família: revisão integrativa. Rev Panam Salud Pública 2014; 35:144-9.

36. Ministério da Saúde. Política Nacional de Humanização da Atenção e da Gestão do SUS. Brasília: Ministério da Saúde; 2006.

37. Edmond KM, Yousufi K, Anwari Z, Sadat SM, Staniczai SM, Higgins-Steele A, et al. Can community health worker home visiting improve care-seeking and maternal and newborn care practices in fragile states such as Afghanistan? BMC Med 2018; 16:106.

38. Garcia ACP, Andrade MAC, Lima EFA, Rocha EMS, Lima RCD. Melhoria do acesso e da qualidade da atenção básica: perspectiva dos Agentes Comunitários de Saúde. Saúde Debate 2018; 42:606-17. 


\section{Abstract}

The study aimed to evaluate care for women with high-risk pregnancies from the healthcare team's perspective in primary healthcare (PHC) services in the city of São Paulo, Brazil. This is an evaluative study with a qualitative approach that used the open interview technique for data collection from PHC personnel. The data were explored through thematic categories built from the content emerging spontaneously from the interviews, discussed according to Health Work Process Theory. The results evidenced three domains: a "normal work routine", organized from the perspective of exclusively biological practices and in which the management model sometimes fails to allow compliance with the recommended practices; a "referral and counter-referral" system, which sometimes allows more timely interventions, more unique to the pregnant women's needs, but still disconnected from the referral services; and "shared responsibility of the PHC team for the pregnant woman", characterized by flexibilization of the work routine that extends beyond application of clinical protocols, encouraging the building of a bond and favoring qualification of care. The study revealed the need for flexibilization of use of patient care protocols in the work process, which can promote the shared responsibility of PHC teams for care, to adjust it to each pregnant woman's unique needs, beyond the limits of services' organization based on the logic of exclusively biological practices.

High-Risk Pregnancy; Prenatal Care; Primary Health Care; Health Services; Health Evaluation

\section{Resumen}

El presente estudio tuvo como objetivo evaluar la atención a las mujeres durante la gestación de alto riesgo bajo la óptica de quien actúa en los servicios de atención primaria en salud (APS) del municipio de São Paulo. Se trata de una investigación evaluativa de abordaje cualitativo, que utilizó la técnica de la entrevista abierta para la recogida de datos con profesionales de la APS. Los datos se investigaron mediante categorías temáticas, construidas a partir del contenido que emergió, de modo espontáneo, de las declaraciones discutidas a partir de la Teoría del Proceso de Trabajo en Salud. Los resultados evidenciaron tres categorías: una "rutina de trabajo protocolaria", organizada desde la perspectiva de prácticas biologicistas y cuyo modelo de gestión, algunas veces, no permite el cumplimiento de las prácticas preconizadas; un sistema de "referencia y contrarreferencia" que a veces permite intervenciones más oportunas y singulares para las necesidades de las gestantes, sin embargo, todavía está desarticulado respecto a los servicios de referencia; y la "corresponsabilización del equipo de APS del cuidado con la gestante", caracterizado por la flexibilización de la rutina de trabajo, que transciende la aplicación de protocolos clínicos, incentivando la construcción de un vínculo y favoreciendo la cualificación del cuidado. Se desveló la necesidad de flexibilización del uso de protocolos asistenciales en el proceso de trabajo, lo que puede impulsar la corresponsabilización de los equipos de APS por el cuidado, a fin de adecuarlo a las singularidades y necesidades de cada gestante, yendo más allá de los límites de la organización, en cuanto a servicios basados en la lógica de prácticas biologicistas.

Embarazo de Alto Riesgo; Atención Prenatal; Atención Primaria de Salud; Servicios de Salud; Evaluación en Salud
Recebido em 30/Set/2020

Versão final reapresentada em 12/Abr/2021

Aprovado em 26/Abr/2021 